\title{
Quantitative assessment of radiation dose and fractionation effects on normal tissue by utilizing a novel lung fibrosis index model
}

Cheng Zhou ${ }^{1,2,3,4^{*}}$, Bleddyn Jones ${ }^{5}$, Mahmoud Moustafa ${ }^{1,2,3,6}$, Christian Schwager ${ }^{1,2,3}$, Julia Bauer ${ }^{2}$, Bing Yang ${ }^{7}$, Liji Cao ${ }^{8}$, Min Jia ${ }^{9}$, Andrea Mairani ${ }^{2,11}$, Ming Chen ${ }^{10}$, Longhua Chen ${ }^{4}$, Juergen Debus ${ }^{1,2,3}$ and Amir Abdollahi $i^{1,2,3^{*}}$

\begin{abstract}
Background: Normal lung tissue tolerance constitutes a limiting factor in delivering the required dose of radiotherapy to cure thoracic and chest wall malignancies. Radiation-induced lung fibrosis (RILF) is considered a critical determinant for late normal tissue complications. While RILF mouse models are frequently approached e.g., as a single high dose thoracic irradiation to investigate lung fibrosis and candidate modulators, a systematic radiobiological characterization of RILF mouse model is urgently needed to compare relative biological effectiveness (RBE) of particle irradiation with protons, helium-, carbon and oxygen ions now available at HIT. We aimed to study the dose-response relationship and fractionation effect of photon irradiation in development of pulmonary fibrosis in C57BL/6 mouse.

Methods: Lung fibrosis was evaluated 24 weeks after single and fractionated whole thoracic irradiation by quantitative assessment of lung alterations using $C T$. The fibrosis index (FI) was determined based on 3D-segmentation of the lungs considering the two key fibrosis parameters affected by ionizing radiation i.e., a dose/fractionation dependent reduction of the total lung volume and increase of the mean lung density.

Results: The effective dose required to induce $50 \%$ of the maximal possible fibrosis $\left(E D_{50}\right)$ was $14.55 \pm 0.34 G y$ and 27. $7 \pm 1.22 \mathrm{~Gy}$, for single and five- fractions irradiation, respectively. Applying a deterministic model an $\alpha / \beta=4.49 \pm 0.38 \mathrm{~Gy}$ for the late lung radiosensitivity was determined. Intriguingly, we found that a linear-quadratic model could be applied to in-vivo log transformed fibrosis (FI) vs. irradiation doses. The LQ model revealed an $\alpha / \beta$ for lung radiosensitivity of 4. $4879 \mathrm{~Gy}$ for single fraction and 3.9474 for 5 -fractions. Our Fl based data were in good agreement with a meta-analysis of previous lung radiosensitivity data derived from different clinical endpoints and various mouse strains. The effect of fractionation on RILF development was further estimated by the biologically effective dose (BED) model with threshold $\operatorname{BED}\left(B E D_{T r}\right)=30.33 \mathrm{~Gy}$ and $B E D_{E D 50}=61.63 \mathrm{~Gy}$, respectively.

Conclusion: The systematic radiobiological characterization of RILF in the C57BL/6 mouse reported in this study marks an important step towards precise estimation of dose-response for development of lung fibrosis. These radiobiological parameters combined with a large repertoire of genetically engineered C57BL/6 mouse models, build a solid foundation for further biologically individualized risk assessment of RILF and functional RBE prediction on novel of particle qualities.
\end{abstract}

Keywords: Thoracic radiotherapy, Lung fibrosis, Fractionation, BED, a/ $\beta$ ratio

\footnotetext{
* Correspondence: c.zhou@dkfz-Heidelberg.de; a.amir@dkfz-Heidelberg.de

${ }^{1}$ German Cancer Consortium (DKTK), Translational Radiation Oncology,

National Center for Tumor Diseases (NCT) and German Cancer Research

Center (DKFZ), INF 460, 69120 Heidelberg, Germany

Full list of author information is available at the end of the article
} 


\section{Background}

Radiotherapy is an integral component for treatment of thoracic tumors and breast cancer, however, the high sensitivity of normal lung tissue to ionizing radiation (IR) leading to long term sequela such as development of pulmonary fibrosis constitutes a major dose limiting constraint for a curative treatment [1]. More recently, hypofractionated stereotactic body or ablative radiation therapy (SBRT, SABR) is increasingly used for e.g., early stage non-small cell lung cancer (NSCLC) and oligometastatic diseases [2, 3]. SBRT is characterized by intensified deposition of radiation doses into one or few circumscribed regions in a single or few fractions [4]. In NSCLC e.g., a biologically effective dose (BED) of $\geq$ $100 \mathrm{~Gy}$ is aimed in 1-8 fractions (based on tumor localization) with excellent local control rates $[5,6]$. The high focused dose is delivered at the expense of a relatively high prescribed dose to the normal lung tissue. Therefore, several approaches are undertaken to estimate the region at risk for long term lung tissue complications after SABR/SBRT $[7,8]$. To minimize normal tissue toxicity, dose volume histogram (DVH) based evaluation are considered, e.g., mean lung dose (MLD $\leq$ 20Gy) and total lung V20 dose-volume constraints being $\leq 35 \%$ according to the recommendation of National Comprehensive Cancer Network (NCCN v4.2016). These dose-volume constraints are mostly empirically based and lack of rigorous preclinical validation. Therefore, there is an urgent need for a systematic characterization and radiobiological modeling of radiation induced pulmonary fibrosis in experimental- and clinical settings for a better understanding and estimation of lung tolerance to ionizing radiation.

The clinical sequelae of radiation injury consist of a subacute onset of radiation-induced inflammation (pneumonitis) with later activation of the fibrogenesis processes [9, 10]. Preclinical studies of radiation-induced lung toxicity in different mouse models [11-15] has improved our understanding of the pathophysiology of radiation lung toxicity and led to the development of biophysical models [16-22]. Among those classical studies, two physiological parameters were most frequently applied for surrogating lung damages induced by ionizing radiation, breathing rate (breaths per minute, BPM) and the lethality $\left(L D_{50}\right)$. By integrating computer assisted radiology, the present study utilized a novel CT imaging based surrogate, fibrosis index $(F I)$ algorithm for the quantitative assessment of lung fibrosis. C57BL/6 mice is among the most frequently studied experimental models of lung fibrosis [23], however, the key parameters determining the lung tissue radiosensitivity were missing. Our data now provide radiobiological estimates for $\alpha / \beta$ ratio and BEDs for the endpoint of radiation induced pulmonary fibrosis in this important preclinical model.
This work was conducted in frame of German Research Foundation (DFG) "clinical research group heavy ion therapy (KFO-214)" in collaboration between the project TP5 and the central platform (ZP1). It builds the conceptual basis for accurate estimation of relative biological effectiveness (RBE) for carbon ions. Data on RBE variation as a function of fractionation and linear energy transfer (LET) utilizing the here presented frame work are in preparation for publication.

\section{Methods}

\section{Irradiation and animals}

Whole thoracic irradiation was administrated to female C57BL/6 mice (Charles River Breeding Laboratories, MA) aged between 8 and 10 weeks. All animal work was approved and performed in compliance with rules outlined by the local and governmental animal care committee instituted by the German government (Regierungspraesidium, Karlsruhe). Photon irradiation was delivered by a $6 \mathrm{MeV}$ Artist Linac (Siemens, Germany) at a dose rate of $3 \mathrm{~Gy} / \mathrm{min}$. Prior to thoracic irradiation, mice were anaesthetized by an intraperitoneal application of $0.36 \mathrm{ml} /$ $\mathrm{kg}$ Rompun 2\% (Bayer HealthCare) and $0.54 \mathrm{ml} / \mathrm{kg}$ ketamine $10 \%$ (Pfizer). Ten anesthetized mice were placed in a specially constructed Polymethylmethacrylat (PMMA) holder for immobilization and irradiated simultaneously. To ensure full coverage of the lung field with breathing motion and sparing neighboring tissues at the maximum, the irradiation plans were adjusted by anatomical and radiological measurement. Dosimetry was used to confirm the dose uniformity in advance.

\section{Experimental design}

A wide range of dose series were included in the doseescalation trials, single fractions (1-fx) arm of: $0,10.5$, 12.5, 14.5, 17.5, 20 Gy; five fractions (5-fx) arm of: 0, 2, 4, 6, 7, 8.5 Gy per fraction. Fractions were given once a day. Each dose group contained 12 randomly grouped mice. Quantitative CT imaging was performed every 4 weeks post irradiation. Based on our previous experiments week 24 was revealed as a suitable interval after radiation to determine late fibrosis development in terms of radiological, histological findings and lethality [1, 24-26]. Mice with signs of severe dermatitis were sacrificed according to the ethics of local governmental animal care committee.

\section{Assessment of lung fibrosis by computed tomography (CT)}

A clinical PET/CT scanner (Biograph mCT, Siemens) was applied for quantitative CT imaging pre- and postirradiation. The standard protocol employed for the CT portion of PET/CT was as follows: $80 \mathrm{kV}$ with $80 \mathrm{mAs}$, a pitch of $0.6 \mathrm{~mm}$, slice thickness of $0.6 \mathrm{~mm}$ and acquisition 
time of $32 \mathrm{~s}$. X-ray exposure is approximately $4.14 \mathrm{mGy}$ per scan. Images were reconstructed using the filter kernel H50s into a transaxial FOV of $138 \times 138 \mathrm{~mm}^{2}$ as a $512 \times 512$ matrix. Images acquired from the clinical CT scanner were viewed and analyzed in MITK software. The lung tissue density was measured by average Hounsfield unit (HU) intensities. The lung, together with all the microstructures, was thereby segmented using a 3D regional growing algorithm with a lower threshold of $-900 \mathrm{HU}$ and an upper threshold of $-100 \mathrm{HU}$. Trachea and primary bronchi were manually resected upon segmentation. Volume sizes and mean HU values within the segmented area were calculated for quantitative assessment of pulmonary toxicity. The fibrosis index was employed to assess the extent of fibrosis as the major endpoint. Briefly, the FI model is based on two critical parameters derived from CT segmented data: the relative increase in mean lung density $(\Delta H U)$ and decreased lung volume $(\Delta V)$ when compared to the mean of an age-matched reference mice cohort. Biologically, the augmented $\triangle H U$ is an overall representation of collagen deposition and increased cellularity; whereas $\Delta V$ reflects the nature of fibrosis as a restrictive lung disease. The calculation of $F I$ is based on the proposed equation as:

$$
\text { Fibrosis index }(\mathrm{FI})=\sqrt{\Delta \overline{\mathrm{HU} \uparrow} \times \Delta \overline{\mathrm{V} \downarrow}}
$$

The presence of radiation fibrosis at 24 weeks post irradiation was determined at the endpoint using delta $\mathrm{HU}$ and delta $\mathrm{V}$ via the segmentation of the entire lung (Fig. 1). Caution is warranted in the case of combined pulmonary fibrosis and emphysema syndrome (CPFE) or pleural effusions. The mean lung density as well as lung volume based on CT measurements might be biased due to the presence of emphysema (air) or effusions (fluids). A supplemented CT histograms analysis, 'peak position of smoothened histogram (PPSH)' was used for a differential diagnosis (see Additional file 1: Appendix).

\section{Analysis of data}

Single and fractionated FIs data were fitted by a modified probit model derived from Kallman et al. [27] using OriginPro 8.0 and Mathematica Software 9.0.

$$
\mathrm{FI}(\mathrm{D})=\frac{1}{2} \mathrm{~A}\left\{1-\operatorname{erf}\left(\sqrt{\pi} \gamma\left(1-\frac{\mathrm{D}}{\mathrm{ED}_{50}}\right)\right)\right\}
$$

where $A$ is the saturation constant for maximal development of fibrosis measured experimentally to be
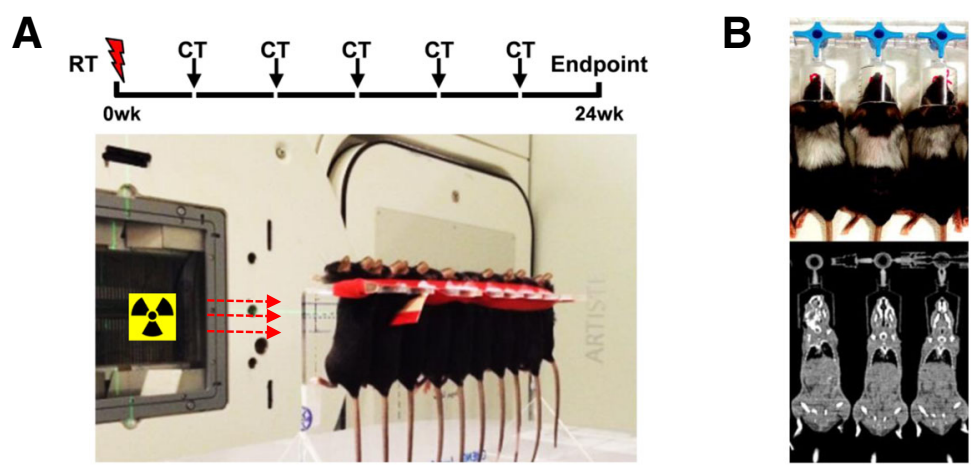

C
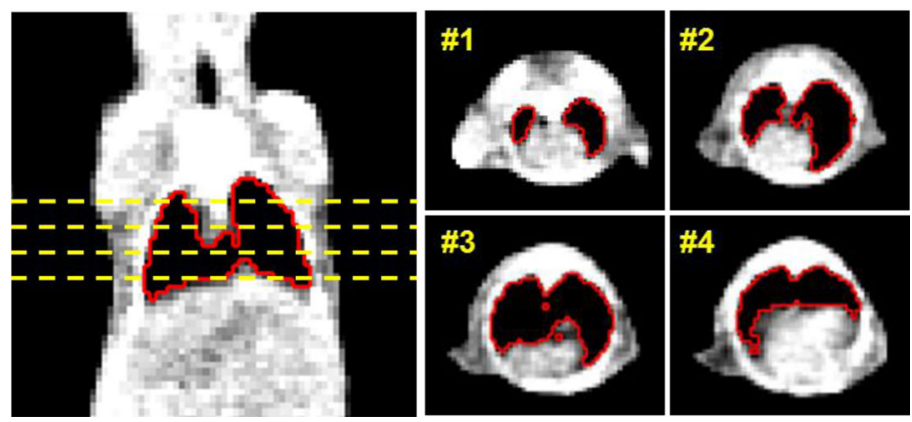

Fig. 1 Overview of the experimental setup. a Simultaneous whole thoracic irradiation with 6 MV Linac (red arrows, anterior-posterior direction of horizontal beam) of up to 10 mice fixed with stretched thoraces in vertical position on a custom holder. Radiotherapy (RT) was followed by longitudinal $C$ imaging every 4 weeks over an overall period of 24 weeks (Endpoint). $\mathbf{b} \subset T$-scan of animals under inhalation anesthesia. Note the thoracic area with gray hairs, lack of melanin, indicating the irradiated region. c Processing of CT-images; the lung area was semi-automatically segmented (red line) and manually curated slice by slice. Total lung volume $\left(\mathrm{mm}^{3}\right)$ and mean lung density (HU) provided the basis for calculation of the lung fibrosis index (FI) as indicated in the methods section. ( $\mathrm{Fl}=$ fibrosis index, $\mathrm{HU}=$ Hounsfield unit, $\mathrm{RT}=$ radiotherapy, $\mathrm{fx}=$ fractionation) 
7.20 (equal to $100 \%$ fibrosis), serving to quantize all FIs. Of note, the FIs versus dose data set used here was continuous rather than event data, hence a deterministic model was applied. Therefore, unlike $E D_{50}$ applied in probabilistic models corresponding to the probability for half $(50 \%)$ of the population (animals) to develop an event, the fibrosis $E D_{50}$ could be here interpretated as the dose where the whole population experiences an average $50 \%$ increase of the $F I(F I=3.60)$ relative to maximum possible effect $(F I=7.20) . \gamma$ is the maximum value of the normalized dose-response gradient. By integrating FIs into the late lung toxicity analysis, eq. (2) was proposed as FI-model for short.

The parameter for fractionation sensitivity $\alpha / \beta$ ratio was derived from the concept of biologically effective dose (BED) [28]. Equivalent BEDs can be achieved by different isoeffective fractionation regimens as:

$$
D_{1}\left(1+\frac{d_{1}}{\alpha / \beta}\right)=D_{2}\left(1+\frac{d_{2}}{\alpha / \beta}\right)
$$

Solving eq. (3), we get $\alpha / \beta$ as:

$$
\frac{\alpha}{\beta}=\frac{D_{2} d_{2}-D_{1} d_{1}}{D_{1}-D_{2}}
$$

Hence, the value of $\alpha / \beta$ can be obtained by two paired dose values, $D_{1}$ and $D_{2}$ giving rise to the same biological effect (namely the equal FI). According to the FI-model, any $D_{X}$ can be determined by the inverse function of eq. (3):

$$
\mathrm{D}^{-1}(F I)=E D_{50}\left[1-\frac{1}{\sqrt{\pi} \gamma} \operatorname{erf}^{-1}\left(1-\frac{2 F I}{\mathrm{~A}}\right)\right]
$$

As a result, for any given $F I$ value $(0.05 \leq F I \leq 7.20)$, we can derive corresponding $D_{1}$ and $D_{2}$ values with reference to single and five fractionation schedules. The estimation of $\alpha / \beta$ was eventually made by referring to eq. (4) within the effective range of FIs.

The FIs data was also analysed as a function of BED doses by logistic regression as:

$$
\mathrm{FI}(\mathrm{BED})=\mathrm{A} 2+\frac{(A 1-A 2)}{\left[1+\left((B E D / k)^{p}\right)\right]}
$$

The threshold of BED to initiating fibrosis $\left(B E D_{T r}\right)$ was defined mathematically as the maximum curvature of the curve; whereas the cut-off dose was derived from the maximum slope.

The simulated data of $\alpha / \beta$ ratios as well as iso-effect doses were fitted with an exponential decay function. For a determination of radiobiological parameters (i.e., $\alpha, \beta, \alpha / \beta)$, the $F I$ data were Log transformed and fitted to the linear-quadratic (LQ) model [18].

\section{Literature review and statistical analysis}

Literature studies were reviewed with reference to radiobiological modeling of late lung damage in mouse models. A total of 13 articles were included and the detailed parameters (i.e. author, publication year, animal, $\alpha / \beta$ ratio, endpoint, follow-up time) were extracted. Forest plots were applied to interpret the values of $\alpha / \beta$ ratio in all studies ( $R$ software v1.5.1). Data is presented as mean \pm SD or otherwise stated. $P<0.05$ is considered as statistically significant.

\section{Results}

Dose-response curves and fibrosis $E D_{50}$

The dose-response curves of RILF using the FI-model is demonstrated (Fig. 2). The radiation effect curve of single dose was much steeper compared to fractionated irradiation $(\gamma=1.64 \pm 0.24,1.41 \pm 0.32$, respectively; Adj. $R^{2}=0.97$, 0.97, respectively). Fibrosis development, as surrogated by $F I$ was markedly enhanced above a threshold dose of $11 \mathrm{~Gy}$. In contrast, normal lung tissue was better spared from radiation injury using fractionated schedules (5-fx). The fibrosis $E D_{50}$ (effective dose for $50 \%$ fibrosis or $F I=3.60$ ) for single- and five- fractions irradiations were identified to be $14.55 \pm 0.34$ Gy and $27.7 \pm 1.22$ Gy, respectively. This indicates an elevated tolerance of normal lung tissue to fractionated photons exposure, in that increasing physical doses were required for the same effectiveness.

\section{$\alpha / \beta$, isoeffect curves and threshold BED}

The fractionation sensitivity related parameter, the $\alpha / \beta$ ratio of lung was estimated using biologically effective dose (BED) equations [28]. The obtained value of $\alpha / \beta$ ratio appears to vary with doses, and was found to be

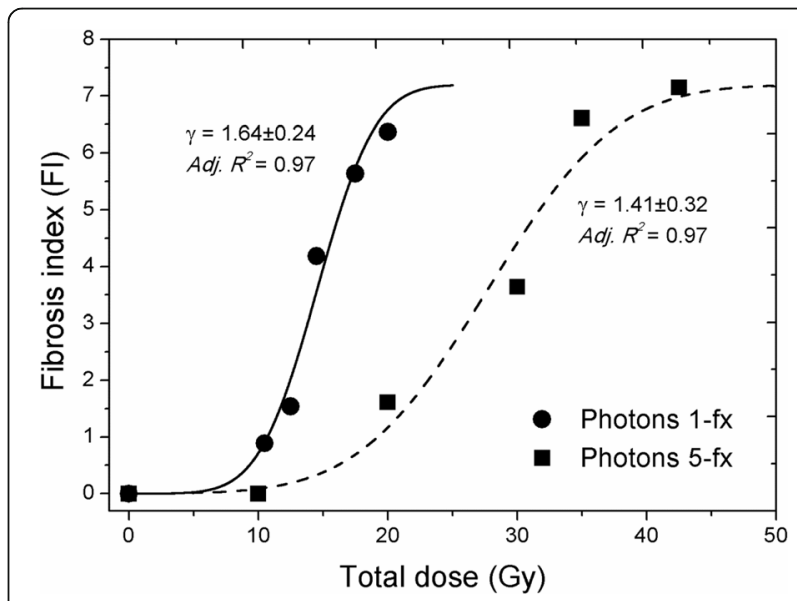

Fig. 2 Dose-response modeling of pulmonary fibrosis induction after single and five fractions of photon irradiations. The sigmoidal curves were plotted based on fibrosis index (FI) (Adjusted $R^{2}=0.972,0.968$ for 1-fx and 5-fx curve, respectively). Single fraction photon doses are shown as solid circles and five fractionated as solid squares 
$4.49 \pm 0.38$ Gy based on FI-model (Fig. 3a). The $\alpha / \beta$ ratio gradually approaches to $4.26 \mathrm{~Gy}$ (at fibrosis $E D_{75}$ ) and even $4.20 \mathrm{~Gy}$ (at fibrosis $E D_{90}$ ) at severe fibrosis level. According to this analysis, using simulated data by the "direct quantal" method [19], the $\alpha / \beta$ ratio obtained at fibrosis $E D_{50}$ was estimated to be $4.38 \mathrm{~Gy}$ (Additional file 1: Figure S1). The iso-effect dose curve with reference to the median, first and third quartiles of the $\alpha / \beta$ ratios are provided (Fig. 3b).

On the other hand, by fitting to LQ model, the principle radiobiological parameters, $\alpha / \beta, \alpha, \beta$ were also obtained approximately as $\alpha / \beta=4.4879$ Gy, $\alpha=0.0480 \mathrm{~Gy}^{-1}$ and $\beta=0.0107 \mathrm{~Gy}^{-2}$ for single dose and $\alpha / \beta=3.9474$ Gy, $\alpha=0.0150 \mathrm{~Gy}^{-1}$ and $\beta=0.0038 \mathrm{~Gy}^{-2}$ for 5 fractionated irradiation (Fig. 4).

BEDs with respect to all irradiated doses were predicted based on the derived $\alpha / \beta$ of 4.49 Gy. The doseresponse relationship between fibrosis development and BEDs is shown (Fig. 5). The threshold BED $\left(B E D_{T r}\right)$ to
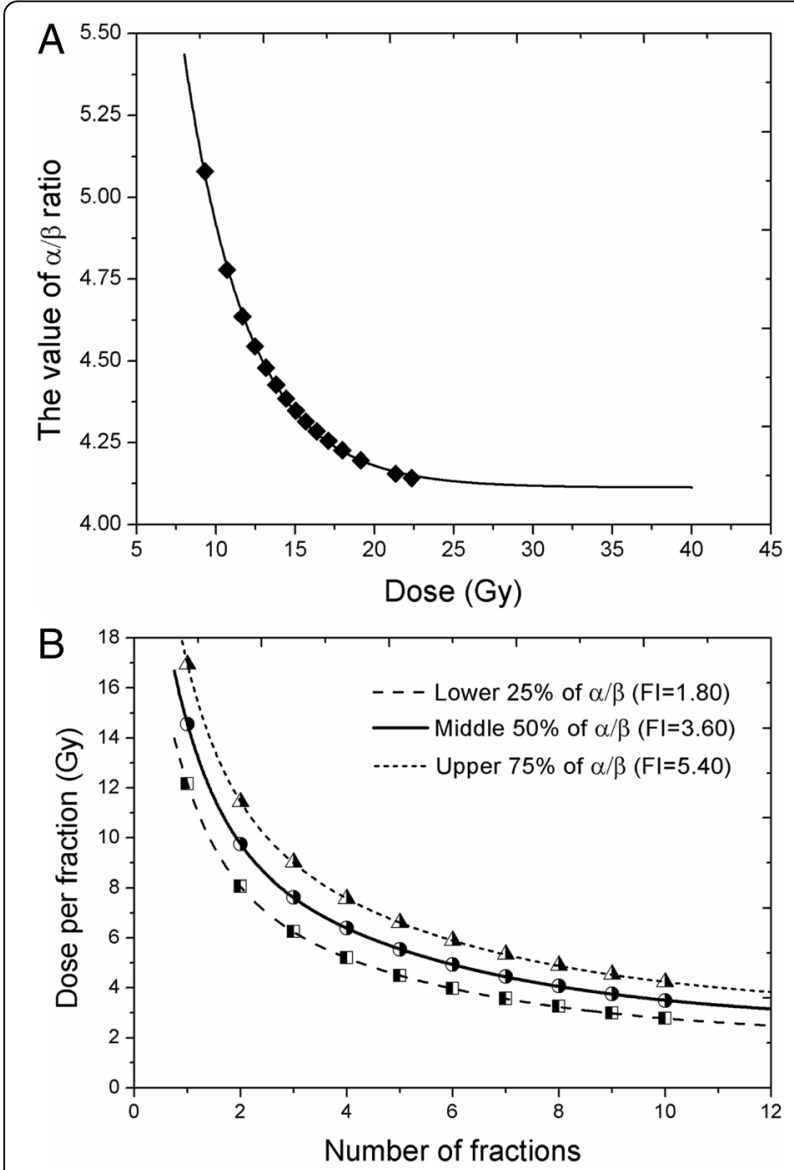

Fig. 3 Simulation based estimation of alpha/beta ratio. Estimates of radiobiological parameters according to simulated iso-effect data revealed that the value of $\alpha / \beta$ ratio for normal lung tissues varies with irradiated doses. a Dose per fraction is plotted against the number of fractions $(\mathbf{b})$. Isoeffect curves with reference to the median, first and third quartiles of $\alpha / \beta$ ratios are shown

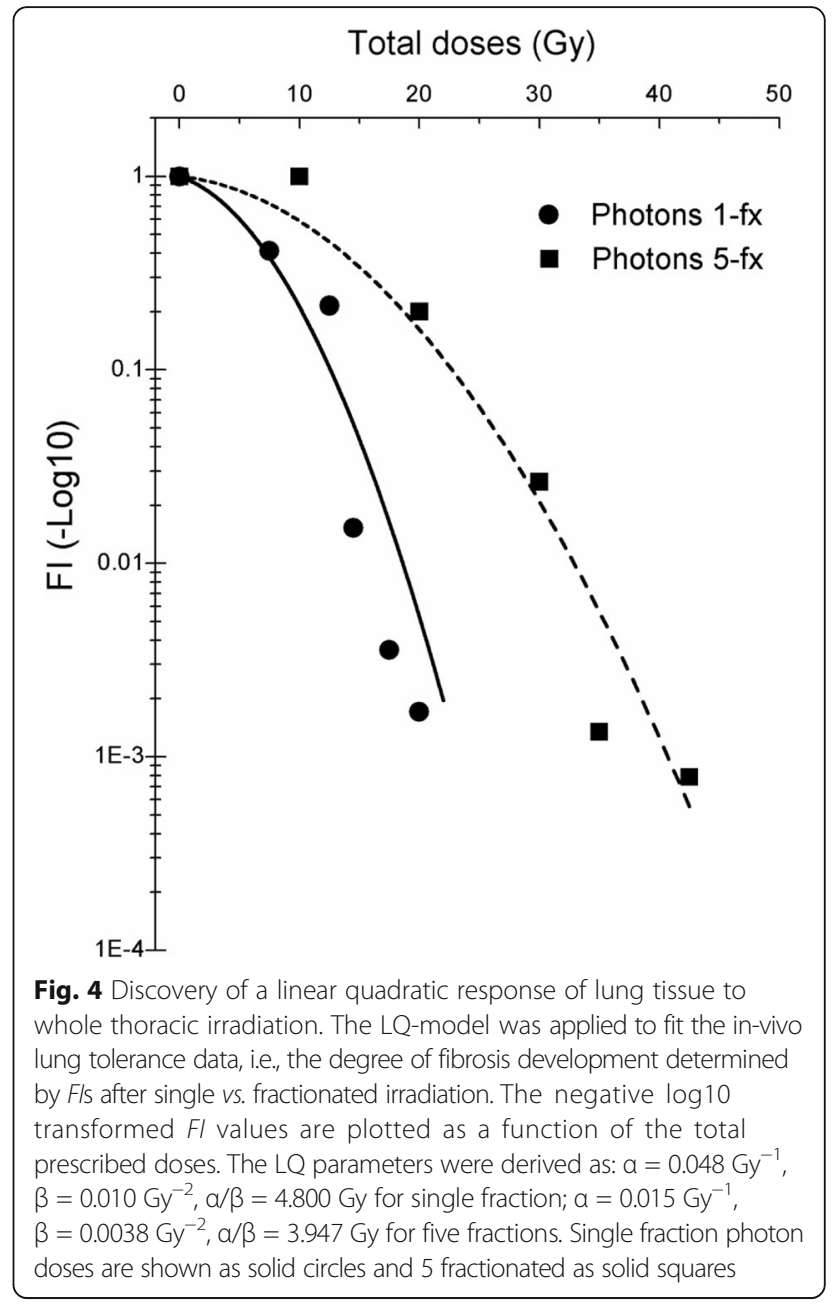

trigger or initiate lung fibrosis was identified as 30.33 Gy. The cut-off BED dose was 54.23 Gy. The $B E D_{E D 50}$ (BED results in $50 \%$ of fibrosis) was determined as $61.63 \mathrm{~Gy}$.

\section{Systematic review of lung $\alpha / \beta$ ratios}

A meta-analysis of experimentally derived lung $\alpha / \beta$ ratios from published studies is shown as a forest plot (Fig. 6). Detailed characteristics of the 13 studies included are provided (Additional file 1: Table S1) $[15,16,21,22,29-37]$. The value of $\alpha / \beta$ ratio may vary with different functional assays, endpoints, follow-up time and biophysical models. The $\alpha / \beta$ ratio discovered by the $F I$-model in our study was consistent with the estimated $\alpha / \beta$ of $4.38 \pm 1.06$ derived from this pooled analysis. Given that different physiological parameters were utilized in the reports included in the meta-analysis, e.g., breath rate and $L D_{50}$, the high agreement of the $\alpha / \beta$ values underscores the robustness of our CT based FI-model to assess lung radiosensitivity. 


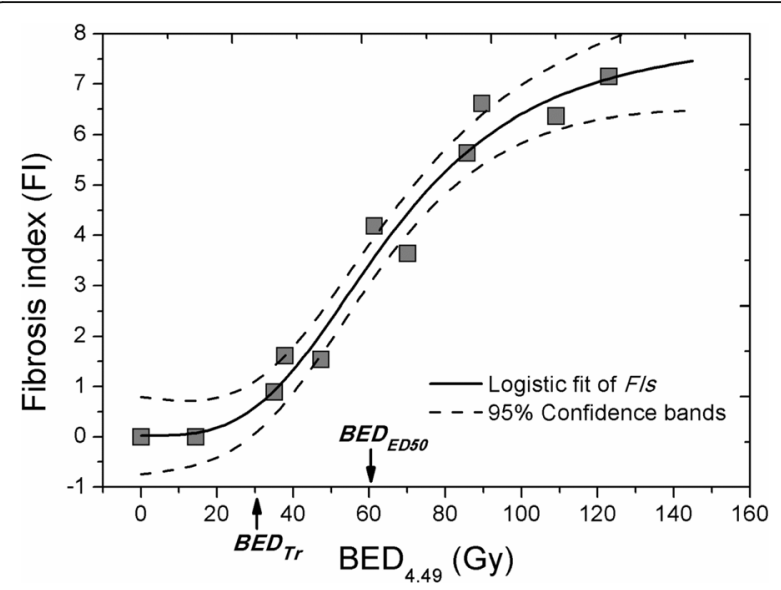

Fig. 5 Biologically effective dose (BED) based dose-response modeling of radiation-induced lung fibrosis. The fibrosis index is plotted against the biological effective doses (BEDs) $\left(\right.$ Adj. $R^{2}=0.959,95 \%$ confidence interval was given within dot lines). Threshold BED (BED $T_{T r}$ ) as well as BED for developing $50 \%$ of fibrosis $\left(B E D_{E D 50}\right)$ is indicated by the arrows

\section{Discussion}

The high sensitivity of lung tissue to ionizing radiation induced damage constitutes a major obstacle for curative radiotherapy of thoracic tumor. Therefore, a better characterization of radiation induced late effects in lung tissue is of utmost interest for the field of radiotherapy. Lung is featured as a late-responding tissue. In accordance with clinical observations, our data suggest a great sensitivity of late responses to changes in fractional dose. Late toxicity was significantly attenuated after 5fractionated doses and the determined fibrosis index changes could be fitted by the LQ model. Preclinical data are limited in evaluating the late lung toxicity occurred with the intent of deriving isoeffective doses.

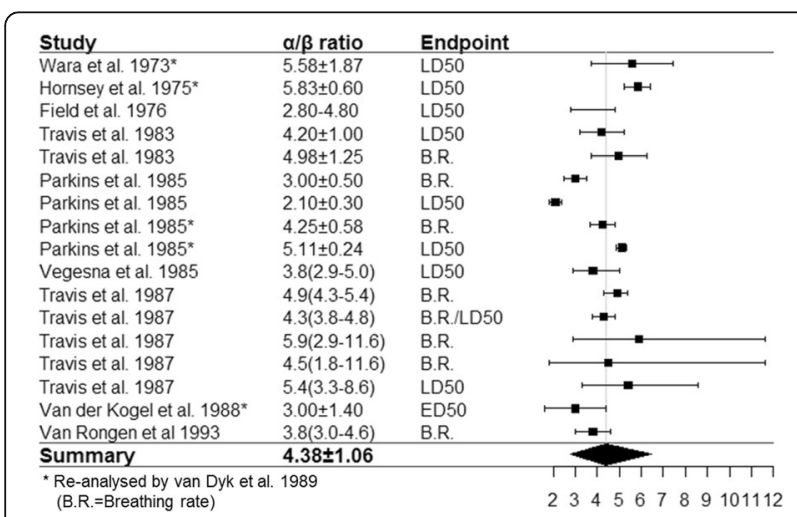

Fig. 6 A systematic review and meta-analysis of $\alpha / \beta$ ratios from previous mouse experiments considering different surrogates for late radiation induced lung damage. An average of $4.38 \pm 1.06$ Gy was estimated from this pooled analysis. The detailed characteristics of included literatures are provided in Additional file 1: Table S1. Data are presented as Mean \pm SE
The isoeffect curves presented here imply that the size of dose per fraction (or the fraction number) plays an essential role in sparing late lung injury. Taken together, our data clearly indicated an unfavourable toxicity profile for delivering a large fractionated dose to normal lung, unless the total dose is carefully selected.

Quantitative estimates of radiobiological characteristics for late phase of pulmonary fibrosis are urgently needed for a better experimental design of translational research in this area. A rough estimate of murine lung $\alpha / \beta$ ranging from 2.4-6.3 Gy was given by Fowler [38]. The $\alpha / \beta$ determined in the present study is $4.49 \pm 0.38 \mathrm{~Gy}$; While at the dose of fibrosis $E D_{50}$, the $\alpha /$ $\beta$ was estimated $\sim 4.38$ Gy. Intriguingly, pooled analysis from the past literature indicates a consensus of $\alpha / \beta$ at $4.38 \pm 1.06$ Gy. Furthermore, the precise dose-response relationship between BED and fibrosis development in mouse was firstly illustrated in this paper. The high comparability of the here presented late lung toxicity data with current empirically derived clinical BED data suggest further exploration of this model including other known key modulators of pulmonary sensitivity to ionizing irradiation such as combination regimens (e.g. radiochemotherapy) or partial volume effects.

Knowledge of the precise value of the average human lung $\alpha / \beta$ ratio is clinically essential, but there is no clear consensus. As reviewed by Bentzen et al., a wide range of $\alpha / \beta$ ratios from 0.9 to 8.5 Gy was reported by different clinical studies based on conventional radiotherapy [39]. In the setting of SBRT, an $\alpha / \beta$ ratio of 3 Gy is most frequently used [40]. Discrepancies between the $\sim 4.4 \alpha / \beta$ ratio obtained under experimentally controlled mouse condition $v s$. different human data may be explained by variability of numerous parameters, i.e., variable lung volumes and positions, cardiac exposure, different surrogates (e.g., pneumonitis or radiographic changes) and species specific responses. It is also possible that the $\alpha / \beta$ ratios could vary with age, cigarette smoking and other air pollution histories.

Among potential limitations of this study could be the restriction of quantitative CT-scan parameters such as lung density to discriminate between lung fibrosis $v s$. inflammation, lung damage induced secondary to cardiac dose, or breathing motions. However, these CT-derived parameters were found to correlate well with a broad spectrum of histopathological and molecular surrogates of lung fibrosis [1, 24, 26]. Further, lethality as a function of irradiation doses was not investigated in this study. Prediction of $\alpha / \beta$ ratio based on LQ model requires a more solid mechanistic basis, as this model so far links the radiation dose with cell survival and repopulation effects.

With advance of particle beam irradiation, the biological evaluation of (sub-) cellular and tissue response to photons, protons and carbon-ions irradiation is urgently needed 
[41-44]. However, preclinical in-vivo comparison of normal tissue effects such as RILF as a function of different radiation qualities are missing. Hence, the proposed FImodel builds a solid bio-math-physical foundation for experimental RBE modeling.

\section{Conclusion}

In conclusion, we have introduced CT imaging based FImodel, providing a quantitative description of radiobiological characteristics as well as the dose-sparing effect of fractionation in a murine model. The $\alpha / \beta$ ratio for fibrosis induction was extracted, in parallel with a pooled analysis from a literature review. Significant sparing of late lung toxicity was illustrated in a fractionated dose regime and threshold "tolerance BED dose" was also determined. Given the spectrum of genetic mouse models available in the here employed C57BL/6 background, our data will impact design and development of personalized normal tissue toxicity estimation and targeted therapeutic interventions. The here reported radiobiological characterization of this model further provides a starting point for determining the RBE for RILF of novel raster scanning proton, helium, carbon and oxygen ions available at HIT.

\section{Additional files}

Additional file 1: Table S1. A list of experimentally derived mouse lung $a / B$ ratios from the literatures with special reference to late lung damage. Data is presented as Mean $\pm \mathrm{SE}$. (B.R. = breathing rate, $F=$ female, $B P M=$ breath per minute, $M=$ male, $d=$ day, $w k .=$ week). Figure $\mathbf{S} 1$. Reciprocal total isoeffect dose for $E D_{50}$ as a function of dose per fraction. The data points were simulated using eq. (3). The $a / \beta$ was obtained as the ratio of the intercept and the slope of the line using the conventional Fe plot. Appendix. $\subset T$ histogram profiling in differential diagnosis of emphysema or pleural effusions. (DOCX $350 \mathrm{~kb}$ )

\section{Abbreviations \\ BED: biologically effective dose; BPM: breaths per minute; CT: computed tomography; DVH: dose volume histogram; Fl: fibrosis index; HU: Hounsfield unit; LET: linear energy transfer; LQ: linear quadratic model; MLD: mean lung dose; NSCLC: non-small cell lung cancer; PMMA: Polymethylmethacrylat; RBE: relative biological effectiveness; RILF: Radiation-induced lung fibrosis; SBRT or SABR: hypofractionated stereotactic body or ablative radiation therapy; V20: \% total lung volume receiving $\geq 20 \mathrm{~Gy}$; a/B: alpha/beta ratio}

\section{Acknowledgements}

We thank Hanning Dai, Physics Institute University of Heidelberg for his support and scientific discussion on data analysis. We thank Barbara Schwager, Claudia Rittmüller and Christine Schmidt for their excellent technical assistance.

\section{Funding}

This work was supported by German Research Council (DFG-KFO214, TP5 and ZP1). It was in part also supported by the Deutsche Krebshilfe (Max-Eder 108,876), intramural grants from National Center for Tumor diseases (NCT 3.02015.22 BioDose and DKTK/DKFZ) and the NSFC (No. 81703166/H2201). The funders had no role in study design, data collection and analysis, decision to publish or preparation of the manuscript.

\section{Availability of data and materials}

The datasets generated during and/or analysed during the current study are available from the corresponding author on reasonable request.

\section{Authors' contributions}

$A A$ and $C Z$ designed the study and prepared the manuscript. $C Z$ and $M M$ performed the animal experiments. CZ, MM, CS, JB, LCC and AM collected, analyzed and interpreted the experimental data. $\mathrm{CZ}$ and $\mathrm{MJ}$ did the literature review and meta-analysis. CZ, BJ and BY did the mathematical modeling. BJ, MC, LHC and JD gave important intellectual input and carefully revised the manuscript. All authors read and approved the final manuscript.

\section{Ethics approval and consent to participate}

All animal work was approved and performed in compliance with rules outlined by the local and governmental animal care committee instituted by the German government (Regierungspraesidium, Karlsruhe).

Consent for publication

Not applicable.

\section{Competing interests}

The authors declare that they have no competing interests.

\section{Publisher's Note}

Springer Nature remains neutral with regard to jurisdictional claims in published maps and institutional affiliations.

\section{Author details}

'German Cancer Consortium (DKTK), Translational Radiation Oncology, National Center for Tumor Diseases (NCT) and German Cancer Research Center (DKFZ), INF 460, 69120 Heidelberg, Germany. ${ }^{2}$ Department of Radiation Oncology, Heidelberg lon-Beam Therapy Centre (HIT), University of Heidelberg Medical School, Heidelberg, Germany. ${ }^{3}$ Heidelberg Institute of Radiation Oncology (HIRO), National Center for Radiation research in Oncology (NCRO), Heidelberg, Germany. ${ }^{4}$ Department of Radiation Oncology, Nanfang Hospital, Southern Medical University, Guangzhou, China. ${ }^{5}$ Gray Laboratory, CRUK/MRC Oxford Oncology Institute, Radiation Oncology, University of Oxford, Oxford, UK. ${ }^{6}$ Department of Clinical Pathology, Suez Canal University, Ismailia, Egypt. 'Physics Institute University of Heidelberg, Heidelberg, Germany. ${ }^{8}$ Inviscan SAS, Strasbourg, France. ${ }^{9}$ Division of Clinical Epidemiology and Aging Research, German Cancer Research Center (DKFZ), Heidelberg, Germany. ${ }^{10}$ Zhejiang Key Lab of Radiation Oncology, Zhejiang Cancer Hospital, Hangzhou, China. ${ }^{11}$ Italian National Center for Oncological Hadron Therapy (CNAO), Pavia, Italy.

Received: 16 August 2017 Accepted: 27 October 2017

Published online: 07 November 2017

\section{References}

1. Abdollahi A, Li M, Ping G, Plathow C, Domhan S, Kiessling F, Lee LB, McMahon G, Grone HJ, Lipson KE, Huber PE. Inhibition of plateletderived growth factor signaling attenuates pulmonary fibrosis. J Exp Med. 2005;201:925-35.

2. Rusthoven KE, Kavanagh BD, Burri SH, Chen C, Cardenes H, Chidel MA, Pugh TJ, Kane M, Gaspar LE, Schefter TE. Multi-institutional phase I/II trial of stereotactic body radiation therapy for lung metastases. J Clin Oncol. 2009;27:1579-84

3. Eriguchi T, Takeda A, Sanuki N, Nishimura S, Takagawa Y, Enomoto T, Saek $\mathrm{N}$, Yashiro K, Mizuno T, Aoki Y, et al. Stereotactic body radiotherapy for T3 and T4NOMO non-small cell lung cancer. J Radiat Res. 2016;57:265-72.

4. Popp I, Grosu AL, Niedermann G, Duda DG. Immune modulation by hypofractionated stereotactic radiation therapy: therapeutic implications. Radiother Oncol. 2016;120:185-94.

5. Timmerman R, Paulus R, Galvin J, Michalski J, Straube W, Bradley J, Fakiris A, Bezjak A, Videtic G, Johnstone $D$, et al. Stereotactic body radiation therapy for inoperable early stage lung cancer. JAMA. 2010;303:1070-6.

6. Solda F, Lodge M, Ashley S, Whitington A, Goldstraw P, Brada M. Stereotactic radiotherapy (SABR) for the treatment of primary non-small cell lung cancer; systematic review and comparison with a surgical cohort. Radiother Oncol. 2013;109:1-7. 
7. Defraene G, van Elmpt W, Crijns W, Slagmolen P, De Ruysscher D. CT characteristics allow identification of patient-specific susceptibility for radiation-induced lung damage. Radiother Oncol. 2015;117:29-35.

8. Chaudhuri AA, Binkley MS, Rigdon J, Carter JN, Aggarwal S, Dudley SA, Qian Y, Kumar KA, Hara WY, Gensheimer M, et al. Pre-treatment non-target lung FDG-PET uptake predicts symptomatic radiation pneumonitis following stereotactic ablative radiotherapy (SABR). Radiother Oncol. 2016;119:454-60.

9. Marks L, Yu X, Vujaskovic Z, Smalljr W, Folz R, Anscher M. Radiation-induced lung injury. Semin Radiat Oncol. 2003;13:333-45.

10. Tsoutsou PG, Koukourakis MI. Radiation pneumonitis and fibrosis: mechanisms underlying its pathogenesis and implications for future research. Int J Radiat Oncol Biol Phys. 2006;66:1281-93.

11. Fowler JF, Parkins CS, Denekamp J, Terry NH, Maughan RL, Travis EL. Early and late effects in mouse lung and rectum. Int J Radiat Oncol Biol Phys. 1982;8:2089-93

12. Thames HD, Hendry JH, Moore JV, Ang KK, Travis EL. The high steepness of dose-response curves for late-responding normal tissues. Radiother Oncol. 1989;15:49-53.

13. Thames HD Jr, Rozell ME, Tucker SL, Ang KK, Fisher DR, Travis EL. Direct analysis of quantal radiation response data. Int J Radiat Biol Relat Stud Phys Chem Med. 1986:49:999-1009.

14. Travis EL. Early indicators of radiation injury in the lung: are they useful predictors for late changes? Int J Radiat Oncol Biol Phys. 1980;6:1267-9.

15. Parkins CS, Fowler JF, Maughan RL, Roper MJ. Repair in mouse lung for up to 20 fractions of $X$ rays or neutrons. Br J Radiol. 1985;58:225-41.

16. Wara WM, Phillips TL, Margolis LW, Smith V. Radiation pneumonitis: a new approach to the derivation of time-dose factors. Cancer. 1973;32:547-52.

17. Douglas BG, Fowler JF. The effect of multiple small doses of $x$ rays on skin reactions in the mouse and a basic interpretation. Radiat Res. 1976;66:401-26.

18. Barendsen GW. Dose fractionation, dose rate and iso-effect relationships for normal tissue responses. Int J Radiat Oncol Biol Phys. 1982;8:1981-97.

19. Thames HD, Jr., Withers HR, Peters $\amalg$, Fletcher $\mathrm{GH}$ : Changes in early and late radiation responses with altered dose fractionation: implications for dosesurvival relationships. Int J Radiat Oncol Biol Phys 1982, 8:219-226.

20. Thames HD. An 'incomplete-repair' model for survival after fractionated and continuous irradiations. Int J Radiat Biol Relat Stud Phys Chem Med. 1985;47:319-39.

21. Field SB, Hornsey $\mathrm{S}$, Kutsutani Y. Effects of fractionated irradiation on mouse lung and a phenomenon of slow repair. Br J Radiol. 1976;49:700-7.

22. Van Dyk J, Mah K, Keane TJ. Radiation-induced lung damage: dose-timefractionation considerations. Radiother Oncol. 1989;14:55-69.

23. BM B, Lawson WE, Oury TD, Sisson TH, Raghavendran K, Hogaboam CM. Animal models of fibrotic lung disease. Am J Respir Cell Mol Biol. 2013:49:167-79.

24. Plathow C, Li M, Gong P, Zieher H, Kiessling F, Peschke P, Kauczor HU, Abdollahi A, Huber PE. Computed tomography monitoring of radiationinduced lung fibrosis in mice. Investig Radiol. 2004;39:600-9.

25. Flechsig P, Hartenstein B, Teurich S, Dadrich M, Hauser K, Abdollahi A, Grone $\mathrm{HJ}$, Angel P, Huber PE. Loss of matrix metalloproteinase-13 attenuates murine radiation-induced pulmonary fibrosis. Int J Radiat Oncol Biol Phys. 2010;77:582-90

26. Li M, Abdollahi A, Grone HJ, Lipson KE, Belka C, Huber PE. Late treatment with imatinib mesylate ameliorates radiation-induced lung fibrosis in a mouse model. Radiat Oncol. 2009;4:66.

27. Kallman P, Agren A, Brahme A. Tumour and normal tissue responses to fractionated non-uniform dose delivery. Int J Radiat Biol. 1992;62:249-62.

28. Jones B, Dale RG, Deehan C, Hopkins Kl, Morgan DA. The role of biologically effective dose (BED) in clinical oncology. Clin Oncol (R Coll Radiol). 2001:13:71-81.

29. Hornsey S, Kutsutani Y, Field SB. Damage to mouse lung with fractionated neutrons and $x$ rays. Radiology. 1975;116:171-4.

30. Travis EL, Parkins CS, Down JD, Fowler JF, Thames HD Jr. Repair in mouse lung between multiple small doses of X rays. Radiat Res. 1983;94:326-39.

31. Parkins CS, Fowler JF. Repair in mouse lung of multifraction $X$ rays and neutrons: extension to 40 fractions. Br J Radiol. 1985;58:1097-103.

32. Vegesna $V$, Withers HR, Thames HD Jr, Mason K. Multifraction radiation response of mouse lung. Int J Radiat Biol Relat Stud Phys Chem Med. 1985;47:413-22.

33. Travis EL, Thames HD, Watkins $T L$, Kiss I. The kinetics of repair in mouse lung after fractionated irradiation. Int J Radiat Biol Relat Stud Phys Chem Med. 1987;52:903-19.
34. Travis EL, Curtis SB, Howard J, Fowler JF. Repair but not potentiation observed in mouse lung irradiated with neon ions. Radiat Res. 1987;112:500-7.

35. van der Kogel AJ, Martin JC, Smith AR, Raju MR. Late effects of fractionated pi-mesons compared to X rays on mouse lung. Int J Radiat Oncol Biol Phys. 1988;14:1175-84.

36. van Rongen E, Thames HD Jr, Travis EL. Recovery from radiation damage in mouse lung: interpretation in terms of two rates of repair. Radiat Res. 1993;133:225-33.

37. Van Dyk J, Keane TJ. Determination of parameters for the linear-quadratic model for radiation-induced lung damage. Int J Radiat Oncol Biol Phys. 1989;17:695.

38. Fowler JF. Fractionated radiation therapy after Strandqvist. Acta Radiol Oncol. 1984;23:209-16.

39. Bentzen SM, Skoczylas JZ, Bernier J. Quantitative clinical radiobiology of early and late lung reactions. Int J Radiat Biol. 2000;76:453-62

40. Fowler JF, Tome WA, Fenwick JD, Mehta MP. A challenge to traditional radiation oncology. Int J Radiat Oncol Biol Phys. 2004;60:1241-56.

41. Chiblak S, Tang Z, Campos B, Gal Z, Unterberg A, Debus J, Herold-Mende C, Abdollahi A. Radiosensitivity of patient-derived Glioma stem cell 3dimensional cultures to photon, proton, and carbon irradiation. Int J Radiat Oncol Biol Phys. 2016;95:112-9.

42. Winter M, Dokic I, Schlegel J, Warnken U, Debus J, Abdollahi A, Schnolzer M. Deciphering the acute cellular phosphoproteome response to irradiation with X-rays, protons and carbon ions. Mol Cell Proteomics. 2017.

43. Niklas M, Abdollahi A, Akselrod MS, Debus J, Jakel O, Greilich S. Subcellular spatial correlation of particle traversal and biological response in clinical ion beams. Int J Radiat Oncol Biol Phys. 2013;87:1141-7.

44. Dokic I, Mairani A, Niklas M, Zimmermann F, Chaudhri N, Krunic D, Tessonnier T, Ferrari A, Parodi K, Jakel O, et al. Next generation multi-scale biophysical characterization of high precision cancer particle radiotherapy using clinical proton, helium-, carbon- and oxygen ion beams. Oncotarget. 2016;7:56676-89.

\section{Submit your next manuscript to BioMed Central and we will help you at every step:}

- We accept pre-submission inquiries

- Our selector tool helps you to find the most relevant journal

- We provide round the clock customer support

- Convenient online submission

- Thorough peer review

- Inclusion in PubMed and all major indexing services

- Maximum visibility for your research

Submit your manuscript at www.biomedcentral.com/submit
Biomed Central 